\title{
Metasurface spatiotemporal dynamics and asymmetric photonic spin-orbit interactions mediated vector-polarization optical chaos
}

\author{
Mingfeng Xu, ${ }^{1,2}$ Fei Zhang $\odot,{ }^{1}$ Mingbo Pu, ${ }^{1,3}$ Xiong Li, ${ }^{1,2,3}$ Xiaoliang Ma, ${ }^{1,3}$ Yinghui Guo $\odot,{ }^{1,3}$ Renyan Zhang $®,{ }^{1,2}$ \\ Minghui Hong $\mathbb{1}^{4, *}$ and Xiangang Luo $\mathbb{1}^{1,3, \dagger}$ \\ ${ }^{1}$ State Key Laboratory of Optical Technologies on Nano-Fabrication and Micro-Engineering, Institute of Optics and Electronics, \\ Chinese Academy of Sciences, Chengdu 610209, China \\ ${ }^{2}$ Division of Frontier Science and Technology, Institute of Optics and Electronics, Chinese Academy of Sciences, Chengdu 610209, China \\ ${ }^{3}$ School of Optoelectronics, University of Chinese Academy of Sciences, Beijing 100049, China \\ ${ }^{4}$ Department of Electrical and Computer Engineering, National University of Singapore, 4 Engineering Drive 3, 117576, Singapore
}

(Received 21 May 2020; accepted 11 February 2021; published 8 March 2021)

\begin{abstract}
We theoretically investigate metasurface spatiotemporal dynamics by introducing laser chaotic dynamics into metasurface domain. The spatiotemporal dynamics takes advantage of both the time-dependent dynamics of optical chaos and the local spatial regulation capability of metasurface. Specifically, an optical spatiotemporal dynamic phenomenon, termed as vector-polarization optical chaos (VPOC), is demonstrated through asymmetric photonic spin-orbit interactions between all-dielectric metalens and chaotic polarization light from the vertical-cavity surface-emitting laser. The VPOC has spatially inhomogeneous polarization distribution with dynamically varied spatial structure and polarization singularity, while the corresponding intensity time series is temporally chaotic, characterized by positive largest Lyapunov exponent and finite correlation dimension. We found that the two nonlinear indices keep invariant for different polarization-resolved intensity time series due to the circular symmetry of metalens. Our results open an avenue for emerging spatiotemporal optical dynamics with metasurface and may find exciting applications in spatiotemporal light control, free-space secure optical communication, and optical chaos integrated chip.
\end{abstract}

DOI: 10.1103/PhysRevResearch.3.013215

\section{INTRODUCTION}

Artificial microstructured and nanostructured metasurfaces could spatially manipulate optical field by light-matter interactions at subwavelength scale and have found tremendous applications in wavefront manipulation, nonlinear optics, and quantum information [1-4]. Active metasurfaces have attracted great attention due to the most important capability of spatiotemporal light control [5,6]. Recently, by introducing an optical frequency comb source, a so-called frequency-gradient metasurface capable of continuous steering and spatiotemporal redirection of light was demonstrated with passive configuration [7]. This pioneering work evidently shows that, utilizing the specifical dynamical characteristics of incident light field, metasurfaces have great potentials for realizing nontrivial functionalities and emerging novel optical spatiotemporal phenomena. However, the study of dynamical interactions between metasurfaces and time-dependent incident light is still seriously lacking.

\footnotetext{
*elehmh@nus.edu.sg

${ }^{\dagger} \operatorname{lxg} @$ ioe.ac.cn

Published by the American Physical Society under the terms of the Creative Commons Attribution 4.0 International license. Further distribution of this work must maintain attribution to the author(s) and the published article's title, journal citation, and DOI.
}

On the other hand, delay-coupled laser could exhibit diversified optical chaos phenomena with time-varying and unpredictable dynamics, such as amplitude chaos, phase chaos, and polarization chaos [8,9]. It has attracted considerable interest for its promising applications in secure optical communication [10], random-number generators [11], and reservoir computing [12]. Recently, polarization chaotic and even hyperchaotic dynamics stemming from nonlinear competition between two polarization modes in the active region have been demonstrated in the vertical-cavity surface-emitting laser (VCSEL) without external modulation [13-16]. In the absence of time-delay information, the scalar polarization chaotic light from solitary VCSEL dramatically enhances the security and randomness of chaotic dynamics and has promising potentials for optical chaos-based applications $[17,18]$. However, the spatiotemporal polarization chaos with spatial inhomogeneous light field has not yet been reported.

In this paper, we present a universal framework for emerging optical spatiotemporal dynamics by combining passive metasurfaces with laser chaos source. We report a spatiotemporal polarization dynamics, vector-polarization optical chaos (VPOC), by regulating the spatial phase of polarization chaotic output of solitary VCSEL with an all-dielectric metalens. To realize vector polarization, the metalens is elaborately designed to perform asymmetric photonic spin-orbit interactions on the incident light with different circular polarization states. We show that the VPOC has spatially inhomogeneous polarization distribution with dynamically varied spatial 


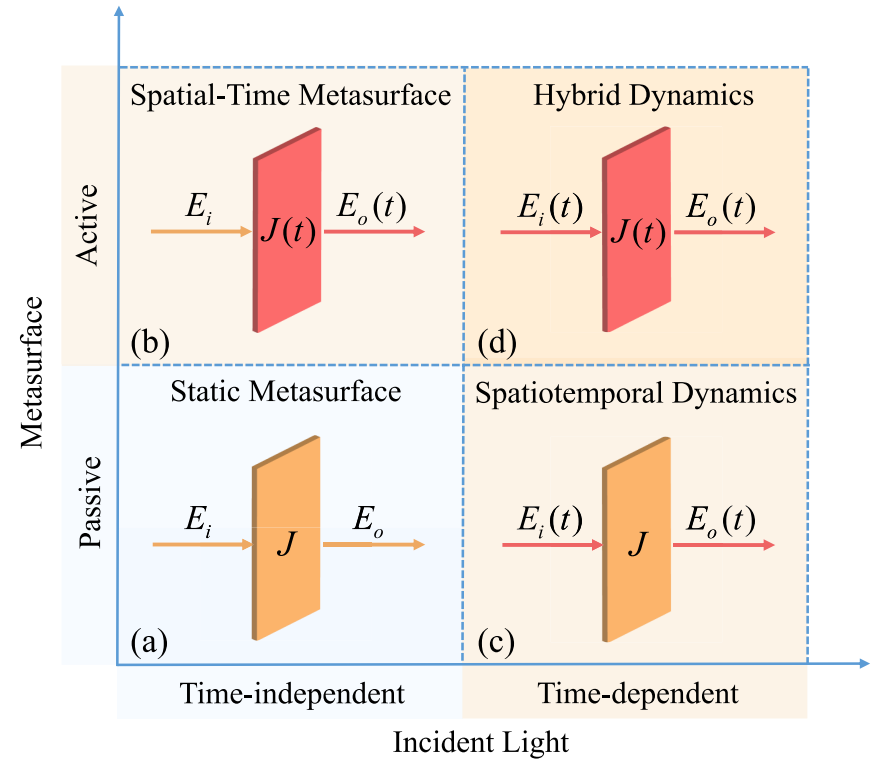

FIG. 1. Paradigms of light-matter interactions between different types of metasurfaces and incident light, from a dynamical perspective.

structure and polarization singularity. Meanwhile, the temporal dynamics of the intensity time series of VPOC is chaotic, demonstrated by the positive largest Lyapunov exponent (LLE) and the finite correlation dimension.

The paper is organized as follows. In Sec. II, we introduce the basic concept of metasurface spatiotemporal dynamics and the principle of generating VPOC. Then in Sec. III, we propose the theoretical model of VPOC based on the asymmetric photonic spin-orbit interactions of metalens. In Sec. IV, we investigate the intensity and polarization dynamics of VPOC. And in Sec. V, the chaotic characteristics are quantitatively analyzed by using the nonlinear time series analysis method. Finally, discussions and conclusions are given in Sec. VI.

\section{METASURFACE SPATIOTEMPORAL DYNAMICS AND VPOC}

As shown in Fig. 1, from a dynamical perspective, the paradigms of light-matter interactions between metasurfaces and incident light can be categorized into four classes, according to different types of incident light (time independent or time dependent) and metasurface (passive or active). The most traditional scenario is static metasurface, wherein both incident light $E_{i}$ and metasurface $J$ are time invariant [Fig. 1(a)]. When the metasurface turns to be active [Fig. 1(b)], the so-called spatial-time metasurface arose, which has attracted considerable interest recently [5]. Here, as presented in Fig. 1(c), we are particularly interested in the third kind of picture called spatiotemporal dynamics, wherein the light-matter interaction occurs between time-dependent incident light and a passive metasurface. Different from the spatial-time metasurface in Fig. 1(b), the dynamical properties of output optical field $E_{o}(t)$ in metasurface dynamics is majorly determined by the incident light $E_{i}(t)$, instead of the metasurface itself. Therefore, with the great capability of optical field regulation, the passive metasurface could serve as an excellent platform

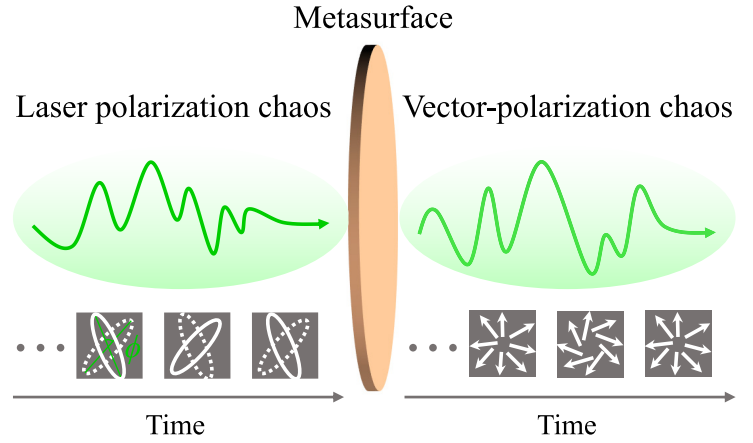

FIG. 2. Concept art of generating VPOC with a solitary VCSEL and a passive metasurface. The two nonorthogonal elliptical polarization states (intersection angle $\phi \approx 40^{\circ}$ ) from VCSEL are chaotically hopped and transformed into two different spirally polarized states by the polarization and wavefront regulation of the metasurface, leading to VOPC.

for exploring novel optical spatiotemporal dynamic behaviors in the all of degrees of freedom of light. Finally, as shown in Fig. 1(d), the universal model of dynamical interaction between metasurface and incident light is hybrid dynamics, which can be described with a time-dependent light $E_{i}(t)$ and an active metasurface $J(t)$.

As illustrated in Fig. 2, we integrate the merits of chaotic light field and passive metasurface to generate VPOC. Specifically, the light emitted from a solitary VCSEL contains two nonorthogonal elliptically polarized chaotic-hopping states with intersection angle $\phi \approx 40^{\circ}$ [13], which is normally incident on a passive metasurface to acquire a spatially inhomogeneous phase change. Here, the chaotic light from VCSEL is assumed as a plane wave when illuminated on metalens. As the chaotic polarization modes are nonorthogonally hopped, the output light from metasurface manifests as randomlike spirally polarized optical field, resulting by the superposition of typical radially polarized (RP) or azimuthally polarized (AP) states. More importantly, the chaotic characteristic is successfully inherited from the scalar polarization chaos by virtue of the fact that the light-matter interactions arising in the metasurface only attach additional phase to incident light, but do not break the time correlations of optical dynamical signals.

\section{THEORETICAL MODEL}

\section{A. VCSEL spin-flip model}

To explore the theoretical model of VPOC, we start with the polarization chaos of solitary VCSEL. Here, the dynamic behaviors of solitary VCSEL are described by the well-known spin-flip model as follows [19]:

$$
\begin{gathered}
\dot{E}_{ \pm}=\kappa(1+i \alpha)(N \pm n-1) E_{ \pm}-\left(i \gamma_{p}+\gamma_{a}\right) E_{\mp}, \\
\dot{N}=-\gamma\left[N-\mu+(N+n)\left|E_{+}\right|^{2}+(N-n)\left|E_{-}\right|^{2}\right], \\
\dot{n}=-\gamma_{s} n-\gamma\left[(N+n)\left|E_{+}\right|^{2}+(N-n)\left|E_{-}\right|^{2}\right],
\end{gathered}
$$

where $E_{ \pm}$denotes slowly varying amplitude of electronic field of right $(+)$ and left $(-)$ circular polarization light from 
VCSEL, $N$ is normalized value of total carrier inversion, $n$ is normalized carrier inversion difference between the two polarization states, $\kappa$ is field decay rate, $\alpha$ is linewidth enhancement factor, $\mu$ is normalized current factor $(\mu=1$ corresponds to threshold current), $\gamma$ is total carrier decay rate, and $\gamma_{s}$ is decay rate of spin-flip processes. $\gamma_{p}$ is linear birefringence and $\gamma_{a}$ is linear dichroism, representing phase anisotropy and amplitude anisotropy, respectively. Moreover, the electric field of horizontal $(X)$ polarization $E_{X}(t)$ and vertical $(Y)$ polarization $E_{Y}(t)$ can be expressed by the circularly polarized light:

$$
E_{X}(t)=\frac{E_{+}+E_{-}}{\sqrt{2}}, \quad E_{Y}(t)=-i \frac{E_{+}-E_{-}}{\sqrt{2}} .
$$

For clarity, the typical laser parameters in our numerical calculations are adopted from Ref. [14]. In fact, deterministic polarization chaos could appear in a solitary VCSEL when the normalized injection current $\mu$ is appropriately regulated, wherein the chaotic attractor successfully emerges in the Poincaré sphere (see Fig. 8 in Appendix A for more details). Moreover, the chaotic dynamics of light emitting from solitary VCSEL is identified by the largest Lyapunov exponent $\lambda_{\max }$ and correlation dimension $D_{2}$ (see Fig. 9 in Appendix A for more details).

\section{B. Asymmetric photonic spin-orbit interactions}

Based on the polarization chaotic light emitting from VCSEL, we design a single-layer all-dielectric metalens with high numerical aperture to efficiently map the scalarpolarization chaotic behaviors into VPOC. As shown in Fig. 3(a), the metalens transforms the $X$-polarization $(Y$ polarization) state into RP state (AP state) and focuses it into a focal solid spot (doughnut-shaped optical spot). The structural materials and geometrical parameters of typical hexagonal unit cell with a lattice constant of $p$ are indicated in Fig. 3(b), where an elliptical silicon post is imposed on a layer of silica substrate with an orientation of $\theta$. Actually, the size and orientation variations of elliptical silicon post could simultaneously introduce the spin-independent propagation phase and the spin-dependent geometric phase [20,21]. The combination of these two phases is utilized to break the symmetry of photonic spin-orbital interactions in metalens to independently regulate right circularly polarized (RCP) and left circularly polarized (LCP) light, leading to independently transform and focus orthogonal linear polarization (LP) light into different vector beams and focusing spots.

\section{Spatiotemporal electric field distribution}

For the chaotic polarization-hopping light from VCSEL, both the amplitude dynamics and the phase difference dynamics for two orthogonal LP components are chaotic [14]. The electric field output from metalens is a chaotic superposition of RP state and AP state, whose mutually orthogonal property ensures that, for arbitrary spatial location, the polarization behaviors could successfully inherit the chaotic characteristic from either the $X$-polarization incident components or the $Y$-polarization incident components. Therefore, the spatiotemporal electric field distribution $\widetilde{E}\left(x, y, z_{0}, t\right)$ output from metalens at $z_{0}=0 \mu \mathrm{m}$ is described as the superposition of

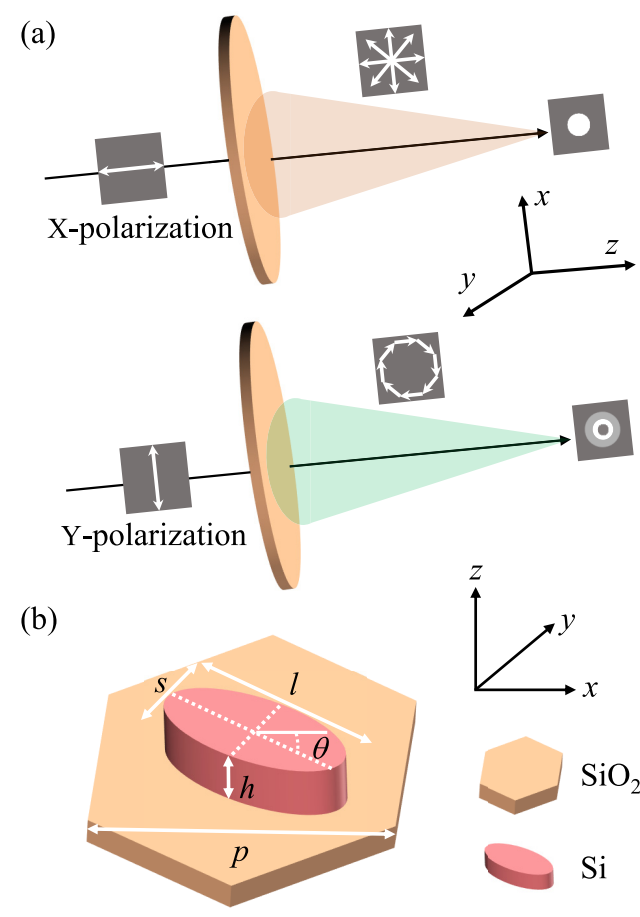

FIG. 3. (a) Schematic diagrams of all-dielectric metalens, which transforms the orthogonal LP light into RP or AP beam and focuses them into different optical spots, respectively. (b) The hexagonal unit cell of all-dielectric metalens with structural materials and geometrical parameters. The combination of propagation phase and geometric phase is designed to break the symmetry of photonic spin-orbit interactions.

RP state $\widetilde{E}_{\mathrm{RP}}\left(x, y, z_{0}, t\right)$ induced by $X$-polarized incident light $E_{X}(t)$ and $\mathrm{AP}$ state $\widetilde{E}_{\mathrm{AP}}\left(x, y, z_{0}, t\right)$ resulted by $Y$-polarized incident light $E_{Y}(t)$ (see Appendix B for more details):

$$
\begin{aligned}
\widetilde{E}\left(x, y, z_{0}, t\right)= & \widetilde{E}_{\mathrm{RP}}\left(x, y, z_{0}, t\right)+\widetilde{E}_{\mathrm{AP}}\left(x, y, z_{0}, t\right) \\
= & \eta \exp \left(-i k \sqrt{x^{2}+y^{2}+f^{2}}\right) \\
& \times\left[\begin{array}{c}
\cos \alpha \cos \varphi E_{X}(t)+\sin \varphi E_{Y}(t) \\
\cos \alpha \sin \varphi E_{X}(t)-\cos \varphi E_{Y}(t) \\
\sin \alpha E_{X}(t)
\end{array}\right],
\end{aligned}
$$

where $\eta$ is transmission amplitude, $k=2 \pi / \lambda$ is wave vector in free space $(\lambda=990 \mathrm{~nm}$ is working wavelength of solitary VCSEL), $f$ is focal length of metalens, $\alpha=$ $\arcsin \sqrt{\left(x^{2}+y^{2}\right) /\left(x^{2}+y^{2}+f^{2}\right)}$ is deflection angle for local position, and $\varphi=\arctan (y / x)=2 \theta$ is azimuthal angle. The time-independent incident electric fields $E_{X}(t)$ and $E_{Y}(t)$ are obtained from spin-flip model of VCSEL, namely, Eqs. (1)-(4). The focal length and size of meta-lens are designed to be $3 \mu \mathrm{m}$ and $12 \mu \mathrm{m} \times 12 \mu \mathrm{m}$, respectively. As a result, the final electric field distribution $\widetilde{E}(x, y, z, t)$ of focusing spots of VPOC beam at any plane with $z>z_{0}$ could be calculated by vectorial angular spectrum theory (see Appendix $\mathrm{C}$ for more details). 

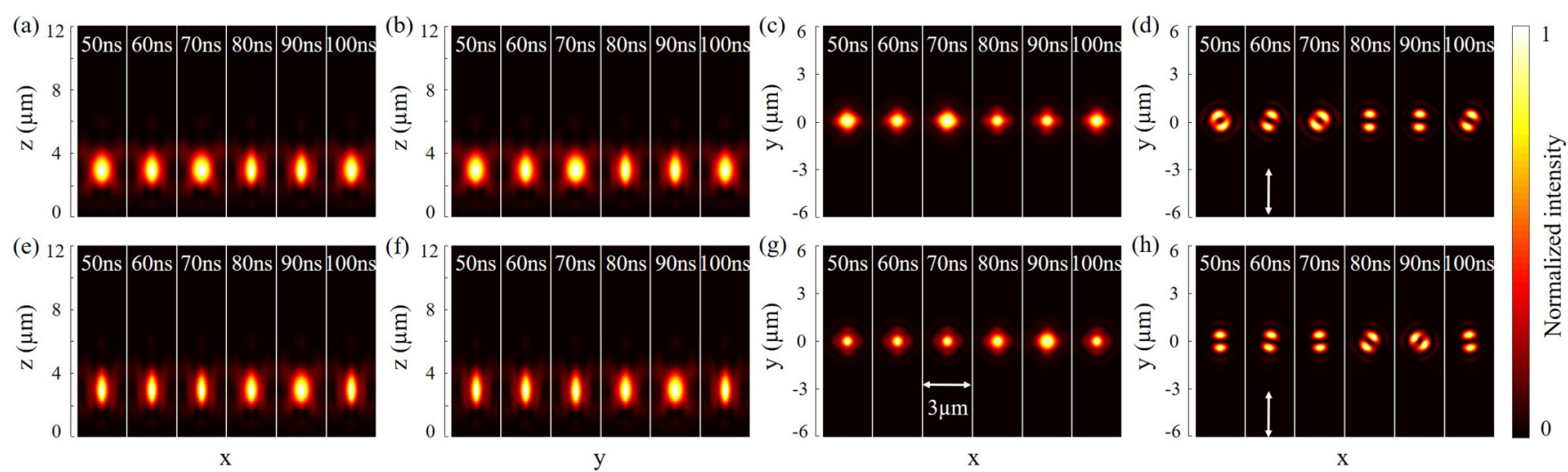

FIG. 4. Dynamical evolution of intensity profiles of VPOC during (50 ns, $100 \mathrm{~ns}$ ) with typical normalized current factor $\mu$ of solitary VCSEL. (a)-(d) $\mu=1.415$. (e)-(h) $\mu=1.423$. From left to right, intensity profile for $x o z$ plane, $y o z$ plane, focal plane $(z=3 \mu \mathrm{m})$, and focal plane with $Y$ polarizer, respectively. The double-sided arrows in the fourth row indicate $Y$ polarizer. Animations of these two VPOC are available in the Supplemental Material [22].

\section{SPATIOTEMPORAL DYNAMICS}

\section{A. Intensity evolution}

In this section, we investigate the intensity evolution of VPOC when VCSEL works in different chaotic regions $(\mu=1.415$ and 1.423). Figures 4(a) $-4(d)$ present the dynamical evolution of intensity profiles of focusing spots in all cross sections when $\mu=1.415$, corresponding to the scenario of Fig. 8(d) where the two single-scroll chaotic attractors emerge in the Poincare sphere. It clearly shows that distinct intensity distributions emerge at different times, in both of $x o z$ plane [Fig. 4(a)] and yoz plane [Fig. 4(b)]. Figure 4(c) shows the evolution of focusing spots in the focal plane $(z=3 \mu \mathrm{m})$, where all the intensity profiles maintain spatially symmetrical distribution. As indicated in Fig. 4(d), the dynamic behaviors become more intuitive from a polarization-resolved perspective, which particularly shows direct evidence of random dynamical property of focusing spots in the vector-polarization space. Figures 4(e)-4(h) present different dynamical evolution behaviors when the VCSEL works in another chaotic region $[\mu=1.423$, corresponding to Fig. 8(e)], all of which exhibit similar random vector-polarization features. It is worth mentioning that the size of focusing solid spots and the $Y$-polarization-resolved dynamic behaviors of these two VPOC are completely distinct, owing to distinct trajectories of the two different chaotic attractors in polarization space.

We note that, in comparison with the optical chaos of VCSEL where the output intensity is polarization dependent, the intensity profiles of VPOC presented in Fig. 4 possess the intrinsic circular symmetry in the polarization space (see Appendix D for details). Therefore, for every particular moment, the polarization-resolved intensity under different polarizers always keeps constant, even though the corresponding spot shapes are completely different. The animations in the Supplemental Material are particularly informative for understanding the dynamical evolution of VPOC [22]. Moreover, the numerical simulation results show that, as expected, the nonchaotic light will not induce the metalens-mediated VPOC and the spatiotemporal polarization dynamics of VPOC emerge when metalens has different focal lengths.
To further investigate the polarization dynamic behaviors of asymmetric photonic spin-orbit interactions mediated VPOC, Fig. 5 presents the polarization-resolved evolution of normalized intensity distribution for $X$ and $Y$ polarizations of focusing optical spots in Fig. 4(h). As shown in Fig. 5(a), the $Y$-polarization component is dominant in comparison with the $X$-polarization component at $50 \mathrm{ns,} \mathrm{resulting} \mathrm{in} \mathrm{a} \mathrm{pair} \mathrm{of}$ beanlike light spots that correspond to $50 \mathrm{~ns}$ in Fig. 4(h). The
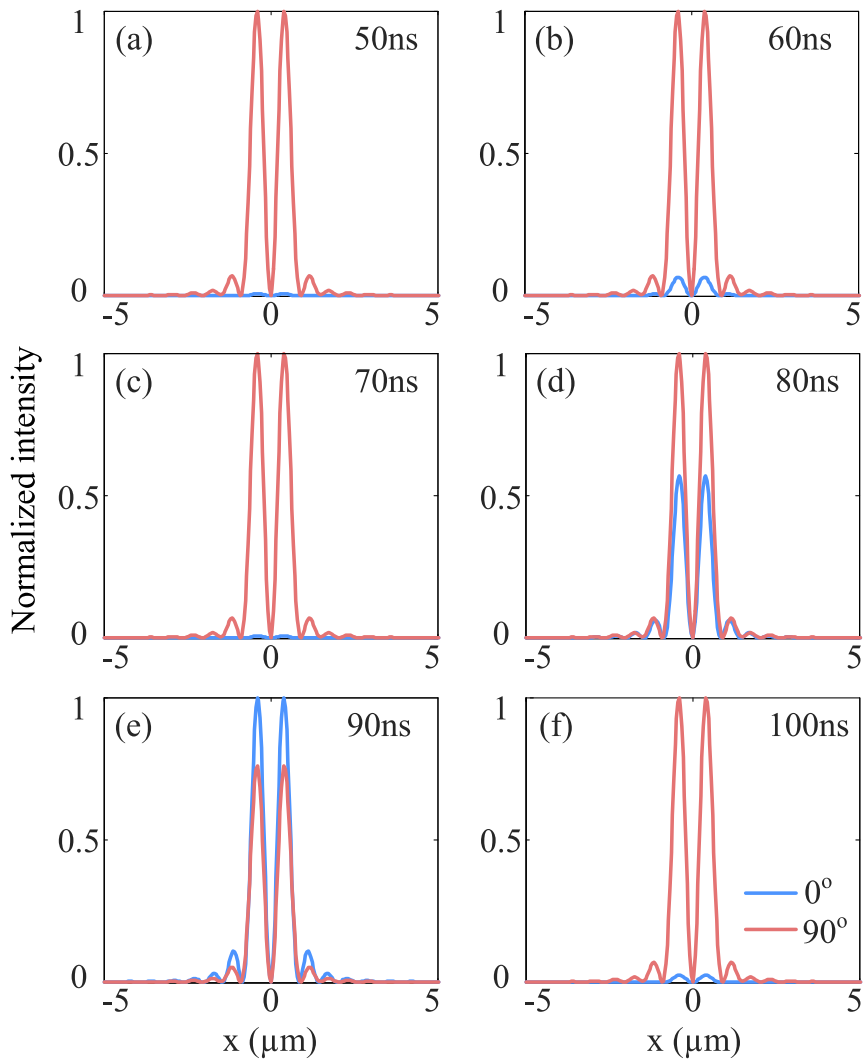

FIG. 5. Dynamical competition between polarization-resolved intensity distribution of VPOC for $X$ - (blue) and $Y$-polarization (red) components when $\mu=1.423$. The polarization-resolved dynamical evolution corresponds to that of Fig. 4(h). 

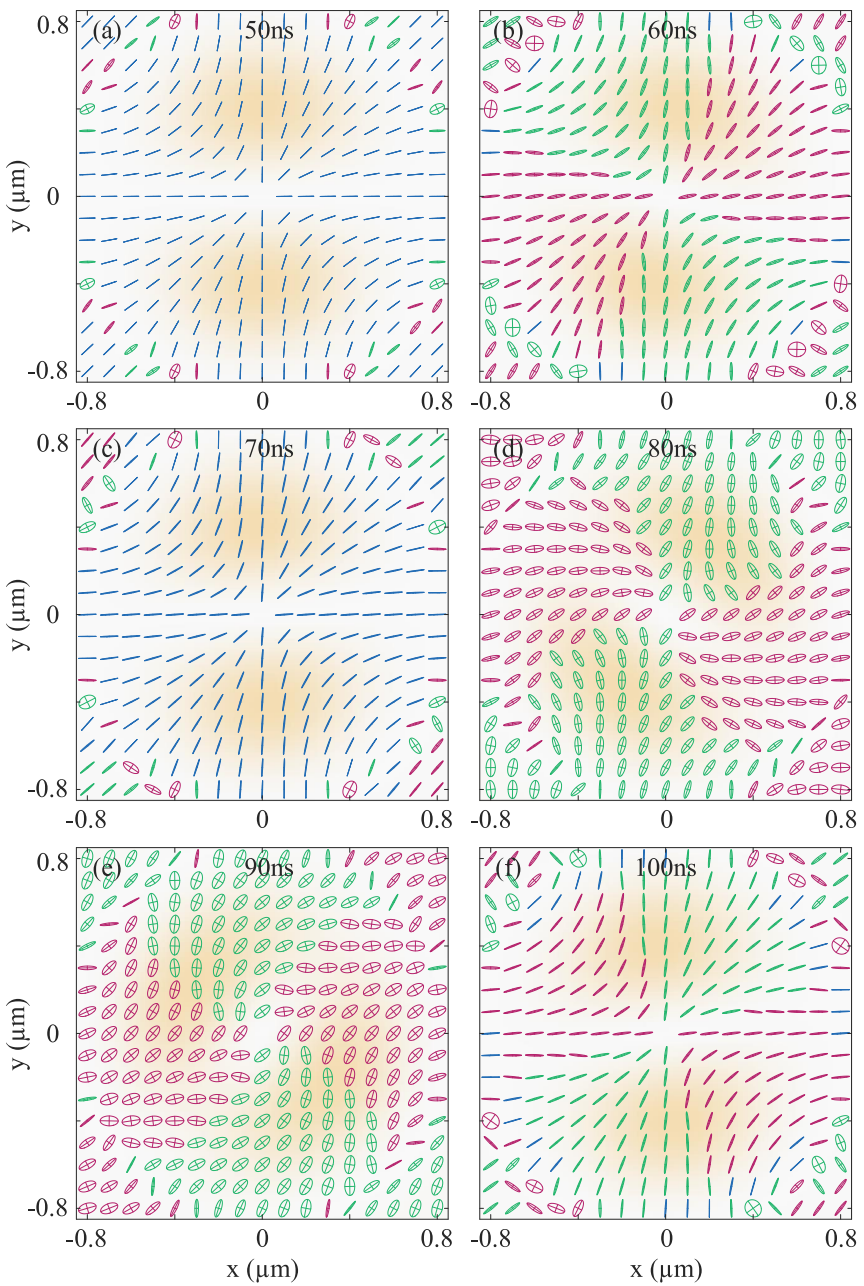

FIG. 6. Intensity (yellow shade) and polarization evolutions of $\widetilde{E}(x, y, z, t)$ in the xoy plane for $\mu=1.423$ and $z=3 \mu \mathrm{m}$, corresponding to Fig. 4(h). The intensity is $Y$-polarization resolved and the major axes of polarization ellipses are normalized. Right-handed $\left(S_{3}>0\right)$, left-handed $\left(S_{3}<0\right)$, and near-linear $\left(S_{3} \simeq 0\right)$ polarizations are represented by red, green, and blue colors.

analogous situations arise in 60,70 , and $100 \mathrm{ns,} \mathrm{correspond-}$ ing to Figs. 5(b), 5(c), and 5(f), respectively. On the other hand, when the two polarization components have similar intensities [Figs. 5(d) and 5(e)], the two beanlike light spots become nonseparated [80 and $90 \mathrm{~ns}$ in Fig. 4(h)]. Therefore, the dynamical competition relationship between $X$ - and $Y$-polarization components in the polarization space induces the abundant dynamic behaviors of focusing optical spots of VPOC.

\section{B. Polarization evolution}

We then explore the dynamical evolution of polarization distribution of the emerging spatiotemporal electric field $\widetilde{E}(x, y, z, t)$ with the corresponding Stokes parameters $\left\{S_{0}, S_{1}, S_{2}, S_{3}\right\}[23,24]$. As shown in Figs. 6(a)-6(f) for $\mu=1.423$ and $z=3 \mu \mathrm{m}$, the spatial polarization distribution patterns have significant spatiotemporal features, manifesting as temporally random varied and spatially inhomogeneous vector-polarization behaviors. Every spatial polarization distribution pattern has circular symmetry as a result of symmetrical superposition of RP light and AP light in the metalens. The polarization singularity resulting from the phase and polarization regulation of metalens appears in the center of polarization space, which is inherently different from the chaotic polarization mode hopping of VCSEL without polarization discontinuities. The polarization ellipses behave as right-handed $\left(S_{3}>0\right)$, left-handed $\left(S_{3}<0\right)$, and near-linear $\left(S_{3} \simeq 0\right.$ ) polarizations at different times. As shown in Figs. 6(a) and 6(c), near-linear polarization states dominate in the transverse plane at 50 and $70 \mathrm{~ns}$. In contrast, elliptically polarized states with opposite chirality completely fill the polarization space at 80 and $90 \mathrm{~ns}$, as shown in Figs. 6(d) and 6(e). Meanwhile, the chirality variation of polarization ellipses appears in different regions for different times due to the interactions between chaotic polarization-hopped light and metalens. Such dynamically varied complex pattern of polarization distribution reveals the spatiotemporal dynamical nature of VPOC.

\section{CHAOTIC CHARACTERISTICS}

In this section, we quantitatively verify the chaotic characteristics of VPOC by using a nonlinear time-series analysis method. Specifically, the largest Lyapunov exponent LLE and correlation dimension $D_{2}$ are estimated from two different intensity time series of focusing spot, i.e., total intensity $I_{\text {total }}$ and polarization-resolved intensity $I_{\xi}$, where $\xi$ is an arbitrary angle between the polarization direction of polarizer and the $X$-polarization direction of light and $\xi \in[0,2 \pi]$. Specifically, for a given $z$ and $t$, the total intensity $I_{\text {total }}$ in transverse plane is calculated from Eq. (C3) as

$$
\begin{aligned}
I_{\text {total }}= & \int_{-\infty}^{+\infty} \int_{-\infty}^{+\infty} I(x, y, z, t) d x d y \\
= & \int_{-\infty}^{+\infty} \int_{-\infty}^{+\infty}\left[\widetilde{E}_{x}^{2}(x, y, z, t)+\widetilde{E}_{y}^{2}(x, y, z, t)\right. \\
& \left.+\widetilde{E}_{z}^{2}(x, y, z, t)\right] d x d y .
\end{aligned}
$$

On the other hand, the $z$ component of electric field gained by the focusing of RP state is discarded when we calculate the polarization-resolved intensity of focusing spot over the transverse plane. It means that the arbitrary polarization angleresolved intensity $I_{\xi}$ is calculated by

$$
\begin{aligned}
I_{\xi}= & \int_{-\infty}^{+\infty} \int_{-\infty}^{+\infty} I_{\xi}(x, y, z, t) d x d y \\
= & \int_{-\infty}^{+\infty} \int_{-\infty}^{+\infty}\left\{\left[\widetilde{E}_{x}(x, y, z, t) \cos (\xi)\right]^{2}\right. \\
& \left.+\left[\widetilde{E}_{y}(x, y, z, t) \sin (\xi)\right]^{2}\right\} d x d y .
\end{aligned}
$$

As shown in Fig. 7(a), the LLE $\lambda_{\max }$ of two intensity time series, respectively, converge to 2.4 and 2.9 , calculated by Wolf's algorithm [25]. Figures 7(b) and 7(c) present the corresponding correlation integral $C_{D}(r)$ of embedding time sequences versus sphere radius $r$, calculated by GrassbergerProcaccia algorithm [26]. The existence of a linear region clearly demonstrates the convergence of $C_{D}(r)$ and the corresponding correlation dimension $D_{2}$ is calculated from the slop of $\ln \left[C_{D}(r)\right]$, where $D_{2} \approx 2.45(\approx 1.46)$ is estimated for $I_{\text {total }}$ 

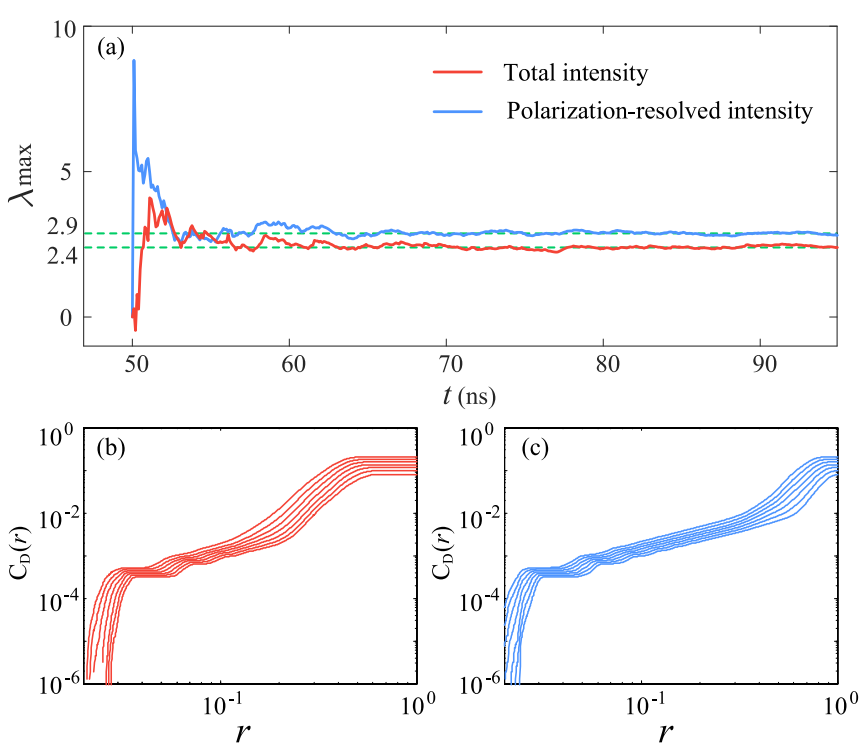

FIG. 7. Chaotic characteristics of VPOC for $\mu=1.423$. (a) LLE for total and polarization-resolved intensity time series of focusing spot. (b), (c) Correlation integral $C_{D}(r)$ versus sphere radius $r$ for total and polarization-resolved intensity time series. The sampling step is 5 ps and the embedding delay is 50 . The embedding dimension from up to down in the $C_{D}(r)$ curves is $m=10-16$.

$\left(I_{\xi}\right)$. Actually, the positive LLE and the finite $D_{2}$ characterize the intrinsically deterministic chaotic behavior of VPOC.

We also note that both LLE and $D_{2}$ between the $I_{\text {total }}$ and the polarization-resolved intensity $I_{\xi}$ are different. In fact, based on the theory of nonlinear time-series analysis [27], Lyapunov exponents and correlation dimensions are only invariant under smooth nonsingular transformations of the state space. Therefore, the deviation of LLE (and $D_{2}$ ) is an inevitable result of the nonsmooth transformation between $I_{\text {total }}$ and $I_{\xi}$, as shown in the comparison between Eqs. (6) and (7). In addition, both the LLE and $D_{2}$ of VOPC are invariant for different polarization-resolved intensity time series owing to the circular symmetry of metalens, which is entirely different from those of the chaotic light of VCSEL (as shown in Fig. 9 in Appendix A). On the other hand, when the VCSEL works in the stable region (for instance, $\mu=1.345$ ), the intensity time series keeps constant all the time and hence the corresponding LLE satisfies $\lambda_{\max } \leqslant 0$, which is totally different from that of VPOC.

\section{DISCUSSION AND CONCLUSION}

In conclusion, we investigate metasurface spatiotemporal dynamics by introducing the chaotic polarization dynamics into metasurface domain. Specifically, we theoretically explore the asymmetric photonic spin-orbit interactions mediated VPOC, which manifests as spatially heterogeneous polarization dynamics. Notably, such spatiotemporal behavior of field polarization is achieved by the temporal dynamics based on the static and space-dependent structure. Therefore, it is completely different from temporal chaos.

The proposed formalism generalizes the spatiotemporal dynamic interactions between artificial subwavelength structures and optical chaos. It enables us to artificially emerge optically spatiotemporal dynamical phenomena in all the degrees of freedom of light, not only limiting in the form of polarization dynamics. For instance, based on the arbitrary spin-to-orbital angular momentum (OAM) conversion technology [28], it is possible to generate chaotic vortex light field wherein the time evolution of OAM state is discrete chaotic. Such optical OAM chaos has potential applications in high-speed secure OAM communication and OAM-based random-number generators.

We notice that spatial chaos [29-31] and instability [32-34] of optical polarization have been extensively studied. In contrast with the traditional spatial polarization chaos, however, the metalens-based configuration makes it possible to artificially regulate the spatially irregular dynamical behaviors of optical fields, which is greatly limited in nonlinear optical medium. Furthermore, integration of VCSEL with metasurface has recently attracted considerable interests for various applications [35-37], given that both VCSEL and metalens have the significant advantages of miniaturization and easy integration. Hence, our VPOC model has great potential to realize chaos-integrated chip, leading to a key step towards to practical applications of optical chaos. Our work opens an exciting adventure for the theories and applications of spatiotemporal optical chaos and offers a perspective for potential applications of metasurface, such as free-space secure optical communication and optical chaos-integrated chip.

\section{ACKNOWLEDGMENT}

The work was supported by National Science Foundation of China (Grants No. 61675208 and No. 61822511) and International Science and Technology Innovation Cooperation Program of Sichuan Province of China (Grant No. 2020YFH0002).

\section{APPENDIX A: POLARIZATION CHAOTIC ATTRACTOR AND CHAOTIC CHARACTERISTICS OF SOLITARY VCSEL}

Figure 8 shows the emergence and evolution of chaotic attractor in the Poincaré sphere, when the normalized injection current $\mu$ is increased from 1.345 to 1.478 . As shown in Fig. 8(a), when $\mu$ is gradually increased around threshold, the
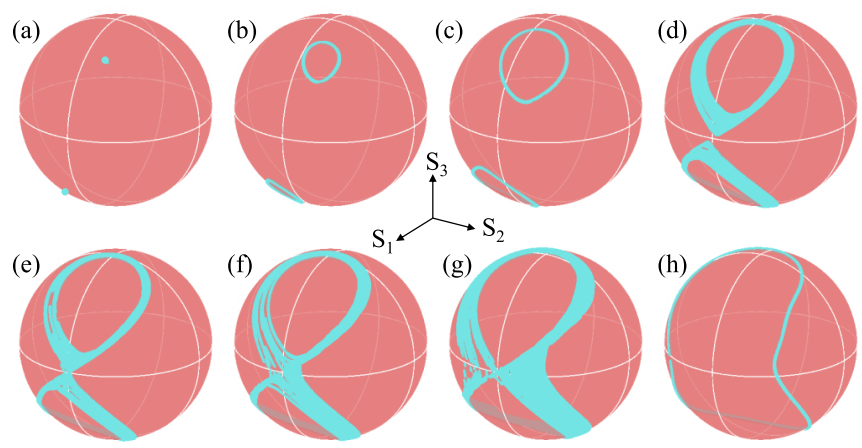

FIG. 8. Dynamical evolution of chaotic attractor in the Poincaré sphere when the normalized current $\mu$ of a solitary quantum dot VCSEL is gradually increased as (a) $\mu=1.345$, (b) $\mu=1.362$, (c) $\mu=1.388$, (d) $\mu=1.415$, (e) $\mu=1.423$, (f) $\mu=1.436$, (g) $\mu=1.465$, and (h) $\mu=1.478 . S_{1}, S_{2}$, and $S_{3}$ are Stokes parameters. 

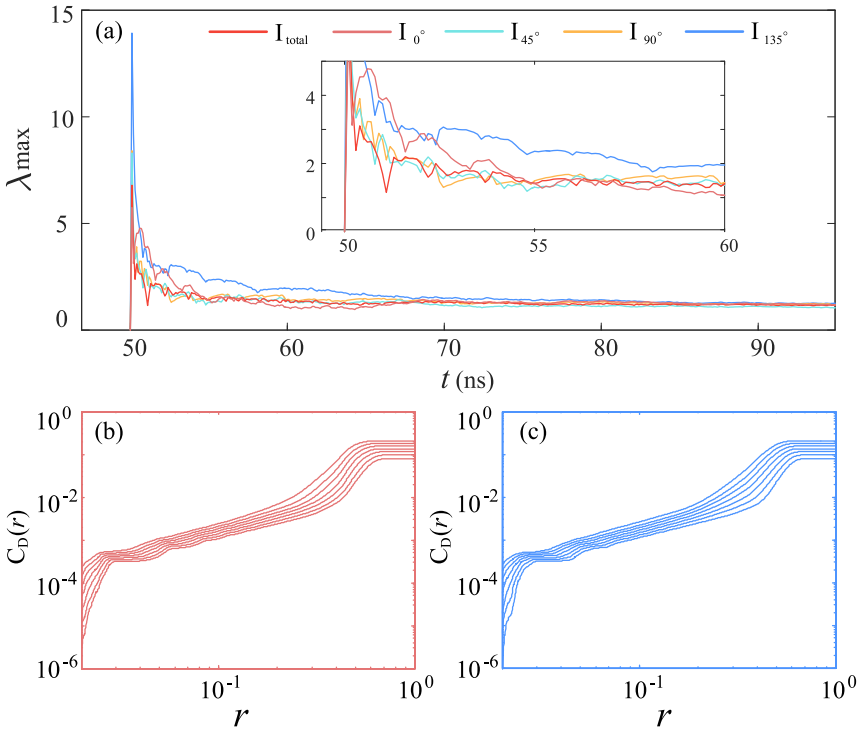

FIG. 9. Chaotic characteristics for solitary VCSEL output. (a) Largest Lyapunov exponent $\lambda_{\max }$ of output power time series of solitary VCSEL with different polarizations when $\mu=1.423$. Correlation integral $C_{D}(r)$ of $X$-polarization output power time series of solitary VCSEL versus sphere radius $r$, for (b) $\mu=1.415$ and (c) $\mu=1.423$. The sampling step is 5 ps and the embedding delay is 50. The embedding dimension from up to down in the $C_{D}(r)$ curves is $m=10-16$.

laser experiences from a stable linearly polarized state to two stable orthogonal elliptically polarized states through a pitchfork bifurcation. As $\mu$ is further increased, the laser emits two unstable nonorthogonal elliptically polarized states, creating two limit cycles in the Poincaré sphere [Fig. 8(b)]. Then, both limit cycles expand [Fig. 8(c)] and there emerge two singlescroll chaotic attractors [Fig. 8(d)] through the cascade of period-doubling bifurcations [13,14], which are centered on the two unstable nonorthogonal elliptically polarized states.

Subsequently, the two chaotic attractors continue to expand and merge into a single double-scroll attractor which manifests like a Lorenz chaotic attractor [Fig. 8(e)], leading to chaotic polarization dynamics. The following evolution shows that, as $\mu$ continuously increases, the trajectories of Lorenztype chaotic attractor cover more and more broad regions in the polarization space of the Poincaré sphere [Figs. 8(f) and $8(\mathrm{~g})$ ]. Finally, a new, bigger limit cycle emerges [Fig. 8(h)] when the value of $\mu$ goes beyond a critical threshold, manifesting as two stable elliptically polarized states for the laser output. Therefore, the trajectory evolutions of chaotic attractor in the Poincaré sphere evidently indicate that solitary VCSEL can exhibit polarization-chaotic dynamics, based on careful choice of injection current.

Furthermore, the chaotic dynamics of solitary VCSEL is identified by largest Lyapunov exponent $\lambda_{\max }$ and correlation dimension $D_{2}$. For the polarization-resolved intensity output, the positive LLE $\lambda_{\max }$ [Fig. 9(a)] and finite correlation dimension $D_{2}$ [Figs. 9(b) and 9(c)] evidently verify the polarization chaotic behaviors of solitary VCSEL output. Note that, for solitary VCSEL chaotic output, different polarizationresolved intensity time series have completely distinct LLE $\lambda_{\text {max }}$.

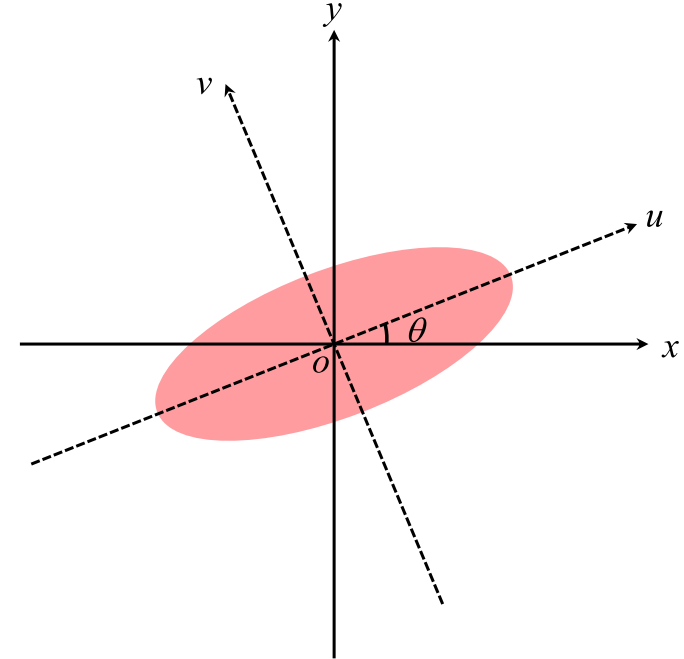

FIG. 10. Localized coordinate system of anisotropic structure.

\section{APPENDIX B: THEORETICAL DERIVATION FOR EQ. (5)}

Without loss of generality, Fig. 10 shows the coordinate relation between the $u-v$ coordinate system for anisotropic structure (elliptical silicon post) and $x-y$ coordinate system for metasurface, where the two coordinate systems have an intersection angle $\theta$. In this scenario, the Jones matrix $\mathbf{M}$ of anisotropic metasurface is described as

$$
\mathbf{M}=\mathbf{R}(-\theta)\left[\begin{array}{cc}
t_{u} & 0 \\
0 & t_{v}
\end{array}\right] \mathbf{R}(\theta),
$$

where $t_{u}$ and $t_{v}$ denote the complex amplitude along with the fast axis and slow axis of anisotropic structure, respectively. $\mathbf{R}(\theta)$ is rotation matrix expressed as

$$
\mathbf{R}(\theta)=\left[\begin{array}{cc}
\cos \theta & \sin \theta \\
-\sin \theta & \cos \theta
\end{array}\right]
$$

Therefore, the general formulation of $\mathbf{M}$ is described as

$$
\mathbf{M}=\left[\begin{array}{ll}
t_{u} \cos ^{2} \theta+t_{v} \sin ^{2} \theta & \left(t_{u}-t_{v}\right) \sin \theta \cos \theta \\
\left(t_{u}-t_{v}\right) \sin \theta \cos \theta & t_{u} \sin ^{2} \theta+t_{v} \cos ^{2} \theta
\end{array}\right] .
$$

For a circular polarization incident light $[1,-i]^{\mathrm{T}}$, its output light from the metasurface is expressed as

$$
\left[\begin{array}{c}
\widetilde{E}_{X} \\
\widetilde{E}_{Y}
\end{array}\right]=\frac{t_{u}+t_{v}}{2}\left[\begin{array}{c}
1 \\
-i
\end{array}\right]+\frac{t_{u}-t_{v}}{2} e^{-i 2 \theta}\left[\begin{array}{l}
1 \\
i
\end{array}\right] .
$$

Generally, we assume that the phase retardation along with the different axis of anisotropic structure are $\beta \pm \delta / 2$, where $\beta$ is propagation phase and $\delta$ is the phase difference between fast axis and slow axis. The corresponding complex amplitudes $t_{u}$ and $t_{v}$ read as

$$
t_{u}=\exp (i \beta-i \delta / 2), \quad t_{u}=\exp (i \beta+i \delta / 2) .
$$

Finally, we get the expression of output light field as

$$
\left[\begin{array}{c}
\widetilde{E}_{X} \\
\widetilde{E}_{Y}
\end{array}\right]=\cos \frac{\delta}{2} e^{i \beta}\left[\begin{array}{c}
1 \\
-i
\end{array}\right]-i \sin \frac{\delta}{2} e^{i(-2 \theta+\beta)}\left[\begin{array}{l}
1 \\
i
\end{array}\right] .
$$

Equation (B6) shows that when a circular polarization light is incident on the metasurface, the output light contains two polarization components. The first component has the same 
polarization as incident light and only carries propagation phase $\beta$. The second component has orthogonal polarization with incident light and, more importantly, it carries both propagation phase $\beta$ and geometric phase $-2 \theta$, yielding the composite phase $\beta-2 \theta$. Obviously, the different output components break the symmetry of spin-orbit interactions of metasurface $[20,21]$.

Theoretically, any LP light can be decomposed into a RCP light and a LCP light and the phase difference between them determines the polarization direction of the LP state. If the incident light is $X$ polarized (the phase difference between RCP and LCP components is zero), it can be rewritten as

$$
E_{\text {in }}=E_{X}=\left[\begin{array}{l}
1 \\
0
\end{array}\right]=\frac{1}{2}\left[\begin{array}{l}
1 \\
i
\end{array}\right]+\frac{1}{2}\left[\begin{array}{c}
1 \\
-i
\end{array}\right]
$$

Therefore, the corresponding output light field $\widetilde{E}$ after the asymmetric spin-orbit interaction is

$$
\begin{aligned}
\widetilde{E} & =\cos \frac{\delta}{2} e^{i \beta}\left[\begin{array}{l}
1 \\
0
\end{array}\right]-\frac{i}{2} \sin \frac{\delta}{2}\left\{e^{i(-2 \theta+\beta)}\left[\begin{array}{l}
1 \\
i
\end{array}\right]+e^{i(2 \theta+\beta)}\left[\begin{array}{c}
1 \\
-i
\end{array}\right]\right\} \\
& =\cos \frac{\delta}{2} e^{i \beta}\left[\begin{array}{l}
1 \\
0
\end{array}\right]-i \sin \frac{\delta}{2} e^{i \beta}\left[\begin{array}{c}
\cos 2 \theta \\
\sin 2 \theta
\end{array}\right]
\end{aligned}
$$

In Eq. (B8), the first term of the right part has the same polarization as incident light, which could be eliminated when $\delta=\pi$. On the other hand, the second term of the right part of Eq. (B8) shows that its polarization and wavefront could be simultaneously modulated by propagation phase $\beta$ and intersection angle $\theta$. Especially, the maximal amplitude is obtained when $\delta=\pi$.

Therefore, it is possible to simultaneously regulate the output polarization and wavefront through reasonable design about the size and orientation of structure cell, which correspond to the propagation phase and geometric phase, respectively. Specifically, we have the following conditions for our metalens:

$$
\begin{aligned}
\delta & =\pi \\
\beta(x, y) & =-k \sqrt{x^{2}+y^{2}+f^{2}}, \\
\theta(x, y) & =\frac{1}{2} \varphi(x, y)=\frac{1}{2} \arctan \left(\frac{y}{x}\right) .
\end{aligned}
$$

Substituting Eqs. (B9) into (B3) and (B5), we can get the Jones matrix $\hat{\mathbf{M}}$ of our metalens as follows:

$$
\hat{\mathbf{M}}=\exp \left(-i k \sqrt{x^{2}+y^{2}+f^{2}}\right)\left[\begin{array}{cc}
\cos \varphi & \sin \varphi \\
\sin \varphi & -\cos \varphi
\end{array}\right] .
$$

Hence, $X$-polarized incident light and $Y$-polarized incident light can be transformed to radially polarized (RP) light and azimuthally polarized (AP) light, respectively. The output electric field can be expressed as

$$
\begin{aligned}
& \widetilde{E}_{\mathrm{RP}}(x, y)=\hat{\mathbf{M}} \cdot\left[\begin{array}{l}
1 \\
0
\end{array}\right]=\exp \left(-i k \sqrt{x^{2}+y^{2}+f^{2}}\right) E_{X 0}\left[\begin{array}{c}
\cos \varphi \\
\sin \varphi
\end{array}\right], \\
& \widetilde{E}_{\mathrm{AP}}(x, y)=\hat{\mathbf{M}} \cdot\left[\begin{array}{l}
0 \\
1
\end{array}\right]=\exp \left(-i k \sqrt{x^{2}+y^{2}+f^{2}}\right) E_{Y 0}\left[\begin{array}{c}
\sin \varphi \\
-\cos \varphi
\end{array}\right],
\end{aligned}
$$

where $E_{X 0}$ and $E_{Y 0}$ are the amplitude of incident $X$-polarized and $Y$-polarized electric field, respectively.

Therefore, for the time-dependent electric field $E(t)$ of laser chaos that can be decomposed into the $X$-polarized incident state $E_{X}(t)$ and $X$-polarized incident state $E_{Y}(t)$, the corresponding output electric fields output from metalens (i.e., $\left.z_{0}=0 \mu \mathrm{m}\right)$ can be described based on the Youngworth's formulas for focusing of cylindrical-vector beams [38]:

$\widetilde{E}_{\mathrm{RP}}\left(x, y, z_{0}, t\right)=\exp \left(-i k \sqrt{x^{2}+y^{2}+f^{2}}\right) E_{X}(t)\left[\begin{array}{cc}\cos \alpha & \cos \varphi \\ \cos \alpha & \sin \varphi \\ \sin \alpha\end{array}\right]$,

$\widetilde{E}_{\mathrm{AP}}\left(x, y, z_{0}, t\right)=\exp \left(-i k \sqrt{x^{2}+y^{2}+f^{2}}\right) E_{Y}(t)\left[\begin{array}{c}\sin \varphi \\ -\cos \varphi \\ 0\end{array}\right]$,

where $\alpha=\arcsin \sqrt{\left(x^{2}+y^{2}\right) /\left(x^{2}+y^{2}+f^{2}\right)}$ denotes deflection angle for local position.

Finally, when we consider the transmission amplitude $\eta$, the spatiotemporal electric field distribution $\widetilde{E}\left(x, y, z_{0}, t\right)$ output from metalens at $z_{0}$ is the superposition of RP state $\widetilde{E}_{\mathrm{RP}}\left(x, y, z_{0}, t\right)$ and $\mathrm{AP}$ state $\widetilde{E}_{\mathrm{AP}}\left(x, y, z_{0}, t\right)$, which reads as Eq. (5).

\section{APPENDIX C: INTENSITY PROFILE CALCULATIONS WITH VECTORIAL ANGULAR SPECTRUM THEORY}

Vectorial angular spectrum theory was used to simulate the vectorial electric field propagation of VPOC beam at any plane with $z>0 \mu \mathrm{m}$ [39]. In all the calculations, the light is assumed to propagate along the $+z$ direction. In fact, $\widetilde{E}\left(x, y, z_{0}, t\right)$ in Eq. (1) represents the the spatiotemporal electric field distribution of VPOC beam at $z_{0}=0 \mu \mathrm{m}$. According to vectorial angular spectrum theory, the vectorial electric field of VPOC beam at $z>z_{0}$ can be expressed as follows [39]:

$$
\begin{aligned}
\widetilde{E}(x, y, z, t)= & {\left[\begin{array}{c}
\widetilde{E}_{x}(x, y, z, t) \\
\widetilde{E}_{y}(x, y, z, t) \\
\widetilde{E}_{z}(x, y, z, t)
\end{array}\right] } \\
= & \int_{-\infty}^{+\infty} \int_{-\infty}^{+\infty}\left[\begin{array}{c}
A_{x, z}(m, n, t) \\
A_{y, z}(m, n, t) \\
\frac{m A_{x, z}(m, n, t)+n A_{y, z}(m, n, t)}{-q}
\end{array}\right] \\
& \times \exp [i 2 \pi(m x+n y)] d m d n .
\end{aligned}
$$

Here, $A_{x, z}(m, n, t)$ and $A_{y, z}(m, n, t)$ are written as

$$
\begin{aligned}
& {\left[\begin{array}{l}
A_{x, z}(m, n, t) \\
A_{y, z}(m, n, t)
\end{array}\right]} \\
& =\exp [i 2 \pi q(m, n) z]\left[\begin{array}{l}
A_{x, z=0}(m, n, t) \\
A_{y, z=0}(m, n, t)
\end{array}\right] \\
& =\exp [i 2 \pi q(m, n) z] \int_{-\infty}^{+\infty} \int_{-\infty}^{+\infty}\left[\begin{array}{l}
\widetilde{E}_{x}\left(x, y, z_{0}, t\right) \\
\widetilde{E}_{y}\left(x, y, z_{0}, t\right)
\end{array}\right] \\
& \quad \times \exp [-i 2 \pi(m x+n y)] d x d y,
\end{aligned}
$$



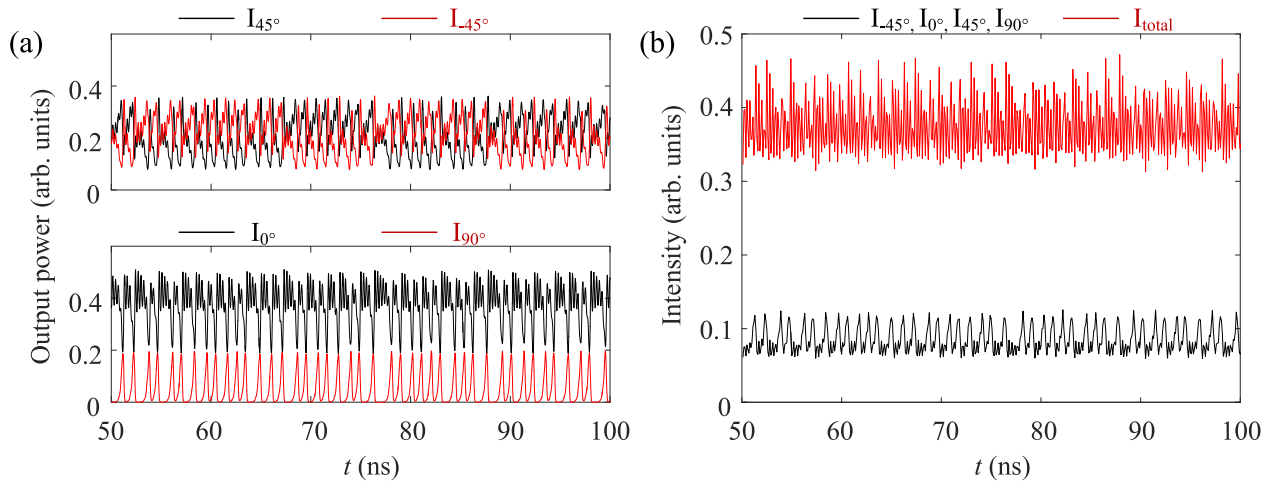

FIG. 11. Comparison of polarization-resolved intensity between VCSEL's chaotic output and VPOC, with normalized injection current $\mu=1.423$. (a) Polarization-resolved output power of solitary VCSEL. Upper row presents the output power in $45^{\circ}$ (black) and $-45^{\circ}$ (red) polarization, and lower row presents the output power in $0^{\circ}$ (black) and $90^{\circ}$ (red) polarization. (b) Polarization-resolved intensity profile of VOPC.

where $m=k_{x} / 2 \pi$ and $n=k_{y} / 2 \pi . k_{x}$ and $k_{y}$ denote the wave numbers along the $x$ and $y$ directions, respectively. $q(m, n)=\sqrt{1 / \lambda^{2}-m^{2}-n^{2}}$ is the spatial frequency. The two initial electric field components, e.g., $\widetilde{E}_{x}\left(x, y, z_{0}, t\right)$ and $\widetilde{E}_{y}\left(x, y, z_{0}, t\right)$, are given in Eq. (5).

Finally, the electric field distribution $\widetilde{E}(x, y, z, t)$ of focusing spots of VPOC beam at any plane with $z>z_{0}$ can be calculated by vectorial angular spectrum theory using Eqs. (C1) and (C2). The intensity profiles in all the figures are calculated as

$$
I(x, y, z, t)=\widetilde{E}_{x}^{2}(x, y, z, t)+\widetilde{E}_{y}^{2}(x, y, z, t)+\widetilde{E}_{z}^{2}(x, y, z, t) .
$$

\section{APPENDIX D: POLARIZATION-RESOLVED INTENSITY ANALYSIS}

As shown in Fig. 11(a), polarization-resolved output power of VCSEL's chaotic output is completely different due to the nonorthogonal property of the two hopped elliptical polarization states. However, as shown in Fig. 11(b), the intensity profiles of VPOC manifest an intrinsic circular symmetry due to the symmetrical phase regulation of metalens in the transversal plane. In comparison with VCSEL's chaotic output, the total intensity of VPOC is well above the corresponding polarization-resolved intensity. Moreover, in the tight focusing scenario, the RP state obtains an additional longitudinal component that could be considerably higher than the transverse component [38].
[1] A. V. Kildishev, A. Boltasseva, and V. M. Shalaev, Planar photonics with metasurfaces, Science 339, 1232009 (2013).

[2] N. Yu and F. Capasso, Flat optics with designer metasurfaces, Nat. Mater. 13, 139 (2014).

[3] X. Luo, D. Tsai, M. Gu, and M. Hong, Extraordinary optical fields in nanostructures: From sub-diffraction-limited optics to sensing and energy conversion, Chem. Soc. Rev. 48, 2458 (2019).

[4] X. Luo, Subwavelength artificial structures: Opening a new era for engineering optics, Adv. Mater. 31, 1804680 (2019).

[5] A. M. Shaltout, V. M. Shalaev, and M. L. Brongersma, Spatiotemporal light control with active metasurfaces, Science $\mathbf{3 6 4}$, eaat3100 (2019).

[6] A. Nemati, Q. Wang, M. Hong, and J. Teng, Tunable and reconfigurable metasurfaces and metadevices, Opto-Electron. Adv. 1, 180009 (2018).

[7] A. M. Shaltout, K. G. Lagoudakis, J. Van De Groep, S. J. Kim, J. Vučković, V. M. Shalaev, and M. L. Brongersma, Spatiotemporal light control with frequency-gradient metasurfaces, Science 365, 374 (2019).

[8] M. C. Soriano, J. García-Ojalvo, C. R. Mirasso, and I. Fischer, Complex photonics: Dynamics and applications of delaycoupled semiconductors lasers, Rev. Mod. Phys. 85, 421 (2013).

[9] M. Sciamanna and K. A. Shore, Physics and applications of laser diode chaos, Nat. Photonics 9, 151 (2015).
[10] A. Argyris, D. Syvridis, L. Larger, V. Annovazzi-Lodi, P. Colet, I. Fischer, J. García-Ojalvo, C. R. Mirasso, L. Pesquera, and K. A. Shore, Chaos-based communications at high bit rates using commercial fibre-optic links, Nature (London) 438, 343 (2005).

[11] A. Uchida, K. Amano, M. Inoue, K. Hirano, S. Naito, H. Someya, I. Oowada, T. Kurashige, M. Shiki, S. Yoshimori, K. Yoshimura, and P. Davis, Fast physical random bit generation with chaotic semiconductor lasers, Nat. Photonics 2, 728 (2008).

[12] L. Appeltant, M. C. Soriano, G. Van der Sande, J. Danckaert, S. Massar, J. Dambre, B. Schrauwen, C. R. Mirasso, and I. Fischer, Information processing using a single dynamical node as complex system, Nat. Commun. 2, 468 (2011);

[13] M. Virte, K. Panajotov, H. Thienpont, and M. Sciamanna, Deterministic polarization chaos from a laser diode, Nat. Photonics 7, 60 (2013).

[14] M. Virte, K. Panajotov, and M. Sciamanna, Bifurcation to nonlinear polarization dynamics and chaos in vertical-cavity surface-emitting laser, Phys. Rev. A 87, 013834 (2013).

[15] C. Bonatto, Hyperchaotic Dynamics for Light Polarization in a Laser Diode, Phys. Rev. Lett. 120, 163902 (2018).

[16] P. Mu, W. Pan, and N. Li, Analysis and characterization of chaos generated by free-running and optically injected VCSELs, Opt. Express 26, 15642 (2018). 
[17] M. Virte, E. Mercier, H. Thienpont, K. Panajotov, and M. Sciamanna, Physical random bit generation from chaotic solitary laser diode, Opt. Express 22, 17271 (2014).

[18] M. Virte, M. Sciamanna, and K. Panajotov, Synchronization of polarization chaos from a free-running VCSEL, Opt. Lett. 41, 4492 (2016).

[19] M. San Miguel, Q. Feng, and J. V. Moloney, Light-polarization dynamics in surface-emitting semiconductor lasers, Phys. Rev. A 52, 1728 (1995).

[20] J. P. B. Mueller, N. A. Rubin, R. C. Devlin, B. Groever, and F. Capasso, Metasurface Polarization Optics: Independent Phase Control of Arbitrary Orthogonal States of Polarization, Phys. Rev. Lett. 118, 113901 (2017).

[21] F. Zhang, M. Pu, X. Li, P. Gao, X. Ma, J. Luo, H. Yu, and X. Luo, All-dielectric metasurfaces for simultaneous giant circular asymmetric transmission and wavefront shaping based on asymmetric photonic spin-orbit interactions, Adv. Funct. Mater. 27, 1704295 (2017).

[22] See Supplemental Material at http://link.aps.org/supplemental/ 10.1103/PhysRevResearch.3.013215 for animations of intensity evolution of VPOC.

[23] M. V. Berry and M. R. Dennis, Polarization singularities in isotropic random vector waves, Proc. R. Soc. Lond. A 457, 141 (2001).

[24] K. Y. Bliokh, M. A. Alonso, and M. R. Dennis, Geometric phases in 2D and 3D polarized fields: Geometrical, dynamical, and topological aspects, Rep. Prog. Phys. 82, 122401 (2019).

[25] A. Wolf, J. B. Swift, H. L. Swinney, and J. A. Vastano, Determining Lyapunov exponents from a time series, Phys. D (Amsterdam) 16, 285 (1985).

[26] P. Grassberger and I. Procaccia, Estimation of the Kolmogorov entropy from a chaotic signal, Phys. Rev. A 28, 2591 (1983).

[27] H. Kantz and T. Schreiber, Nonlinear Time Series Analysis (Cambridge University Press, New York, 2003).

[28] R. C. Devlin, A. Ambrosio, N. A. Rubin, J. P. B. Mueller, and F. Capasso, Arbitrary spin-to-orbital angular momentum conversion of light, Science 358, 896 (2017).
[29] J. Yumoto and K. Otsuka, Frustrated Optical Instability: Self-Induced Periodic and Chaotic Spatial Distribution of Polarization in Nonlinear Optical Media, Phys. Rev. Lett. 54, 1806 (1985).

[30] S. Wabnitz, Spatial Chaos in the Polarization for a Birefringent Optical Fiber with Periodic Coupling, Phys. Rev. Lett. 58, 1415 (1987).

[31] S. Trillo and G. Assanto, Polarization spatial chaos in second-harmonic generation, Opt. Lett. 19, 1825 (1994).

[32] K. Hayata, A. Misawa, and M. Koshiba, Spatial polarization instabilities due to transverse effects in nonlinear guided-wave systems, J. Opt. Soc. Am. B 7, 1268 (1990).

[33] W. Lange, A. Aumann, T. Ackemann, and E. Büthe, Polarization patterns in alkaline vapours, Quantum Semiclass. Opt. 10, R23 (1998).

[34] S. Pitois, G. Millot, and S. Wabnitz, Nonlinear polarization dynamics of counterpropagating waves in an isotropic optical fiber: Theory and experiments, J. Opt. Soc. Am. B 18, 432 (2001).

[35] K. Li, Y. Rao, C. Chase, W. Yang, and C. J. Chang-Hasnain, Monolithic high-contrast metastructure for beam-shaping VCSELs, Optica 5, 10 (2018).

[36] C. A. Curwen, J. L. Reno, and B. S. Williams, Broadband continuous single-mode tuning of a short-cavity quantum-cascade VECSEL, Nat. Photonics 13, 855 (2019).

[37] Y. Xie, P. Ni, Q. Wang, Q. Kan, G. Briere, P. Chen, Z. Zhao, A. Delga, H. Ren, H. Chen, C. Xu, and P. Genevet, Metasurfaceintegrated vertical cavity surface-emitting lasers for programmable directional lasing emissions, Nat. Nanotechnol. 15, 125 (2020).

[38] K. S. Youngworth and T. G. Brown, Focusing of high numerical aperture cylindrical-vector beams, Opt. Express 7, 77 (2000).

[39] M. Pu, X. Li, X. Ma, Y. Wang, Z. Zhao, C. Wang, C. Hu, P. Gao, C. Huang, H. Ren, X. Li, F. Qin, J. Yang, M. Gu, M. Hong, and $\mathrm{X}$. Luo, Catenary optics for achromatic generation of perfect optical angular momentums, Sci. Adv. 1, e1500396 (2015). 